\title{
Cholangiocarcinoma: from molecular biology to treatment
}

\author{
Ana F. Brito ${ }^{1,2,3}$ • Ana M. Abrantes ${ }^{1,2,3} \cdot$ João C. Encarnação ${ }^{1,2}$. \\ José G. Tralhão ${ }^{1,2,4} \cdot$ Maria F. Botelho ${ }^{1,2,3}$
}

Received: 23 September 2015/ Accepted: 25 September 2015

(C) Springer Science+Business Media New York 2015

\begin{abstract}
Cholangiocarcinoma is a rare tumor originating in the bile ducts, which, according to their anatomical location, is classified as intrahepatic, extrahepatic and hilar. Nevertheless, incidence rates have increased markedly in recent decades. With respect to tumor biology, several genetic alterations correlated with resistance to chemotherapy and radiotherapy have been identified. Here, we highlight changes in KRAS and TP53 genes that are normally associated with a more aggressive phenotype. Also IL-6 and some proteins of the BCL-2 family appear to be involved in the resistance that the cholangiocarcinoma presents toward conventional therapies. With regard to diagnosis, tumor markers most commonly used are CEA and CA 19-9, and although its use isolated appears controversial, their combined value has been increasingly advocated. In imaging terms, various methods are needed, such as abdominal ultrasound, computed tomography and cholangiopancreatography. Regarding therapy, surgical modalities are the only ones that offer chance of cure; however, due to late diagnosis, most patients cannot take advantage of them. Thus, the majority of patients are directed to other therapeutic modalities like chemotherapy, which, in this context,
\end{abstract}

Ana F. Brito

anabrito816@gmail.com

1 Biophysics Unit, Faculty of Medicine, University of Coimbra, Pólo III - Pólo das Ciências da Saúde, Azinhaga de Santa Comba, Celas, 3000-548 Coimbra, Portugal

2 Center of Investigation on Environmental, Genetics and Oncobiology (CIMAGO), Faculty of Medicine, University of Coimbra, Coimbra, Portugal

3 CNC.IBILI, Faculty of Medicine, University of Coimbra, Coimbra, Portugal

4 Surgical Department A, CHUC, Coimbra, Portugal assumes a purely palliative role. Thus, it becomes urgent to investigate new therapeutic options for this highly aggressive type of tumor.

Keywords Cholangiocarcinoma $\cdot$ Molecular biology · IL-6 · Diagnosis · Treatment

\section{Cholangiocarcinoma epidemiology}

Cholangiocarcinoma (CC), with origin on the biliary tree cells, is the second most frequent primary liver tumor [1,2]. Anatomically, and according to its location, CC can be classified into intrahepatic, extrahepatic and hilar. The intrahepatic or peripheral $\mathrm{CC}$ is the one that originates in the intrahepatic biliary tree, while extrahepatic CC arises between the ampulla of Vater and the hepatic hilum and hilar $\mathrm{CC}$ emerges due to the confluence of the right and left hepatic channels [1,3-6]. The hilar CCs normally cause premature and obstructive jaundice and when detected are usually small. On the other hand, extrahepatic CCs may be slightly higher although, in most cases, they are detected with a small size. Intrahepatic CCs frequently acquire large dimensions before detection. Despite the differences mentioned, histologically the three CC types are very similar $[3,5,6]$.

Although being considered a rare tumor, the incidence and prevalence of $\mathrm{CC}$ vary markedly worldwide and there are places where it exceeds clearly hepatocellular carcinoma (HCC) incidence. The lowest incidence rates can be found in Australia and the highest in Southeast Asia where this tumor is considered a public health problem [3, 5, 7]. In the northeast of Thailand, the region of the globe with highest incidence of $\mathrm{CC}$, this tumor represents $85 \%$ of primary liver tumors. Also within this country, there are large differences in the incidence of this malignancy from 
region to region. While in the northeast the incidence rates are about 85 new cases per 100,000 habitants, in the north, center and south of the country the incidence rates are only 14.6 per $100,000,14.4$ per 100,000 and 5.7 per 100,000 habitants, respectively [8]. In general, CC incidence has been increasing markedly in Western countries and, although the reason for this increase is not clearly identified, it is known, for example, that there is a correlation between the increased incidence in North America and the migration of Asians to this region [3, 5, 7]. In Western countries, the median age at diagnosis is about 65 years and, with the exception of patients who have previously developed primary sclerosing cholangitis, $\mathrm{CC}$ is rarely diagnosed before the forties. It is also known that 60 to $70 \%$ of cases of CC occur in males [3, 5, 9].

Currently, surgical therapies offer the only chance of cure; however, at the time of diagnosis, the overwhelming majority of patients no longer can benefit from surgical resection and will die between 6 months to a year after diagnosis. Thus, mortality rate almost completely accompanies the incidence rate. Death occurs primarily by hepatic insufficiency and/or infectious complications that go along with advanced biliary obstruction $[3,5]$.

The causes for developing CC are, in most cases, still unknown, considering that the majority of cases occur sporadically; however, there are several conditions associated with biliary tract inflammation and cholestasis that have been identified as risk factors for the development of this neoplasm $[3,5,10,11]$. Primary sclerosing cholangitis (PSC) is considered the most common risk factor, and the development of $\mathrm{CC}$ in patients suffering from this condition usually occurs in the first two and a half years after diagnosis. Thus, it is extremely important to watch over patients continuously diagnosed with PSC [3, 5, 11]. Liver infection by parasites, mainly Opisthorchis viverrini and Clonorchis sinensis species, has also been described as a risk factor for $\mathrm{CC}$ development. This type of infection is most evident in regions of Southeast Asia such as Thailand where poorly cooked fish intake is a common practice and where a high endemicity is reported. Currently, infection with these worms is considered the main reason why there is so much disparity in the incidence of this neoplasia in Thailand [3, 5, 11]. Another risk factor is the biliary lithiasis, usually correlated with chronic biliary infections and most commonly found in Asia than in Western countries. At this point, it is assumed that about $10 \%$ of the patients with this condition will subsequently develop CC $[3,5,11]$.

It is also known that the congenital abnormalities of the bile ducts, such as the cysts of the congenital bile ducts and Caroli's disease, are risk factors for the development of this neoplasia, having these patients an increased risk of 10 to $15 \%$ for $\mathrm{CC}$ development [3, 5, 11]. More recently, infection with hepatitis $\mathrm{B}$ and $\mathrm{C}$ virus has also been considered risk factors for CC development, which may, in part, explain the increased incidence of this neoplasia over the last decades. Several other factors have been associated with the CC development, and this list includes, for example, alcoholic cirrhosis, smoking, obesity and nitrosamines intake $[5,11,12]$.

\section{Molecular and genetic alterations}

A wide range of oncogenic mutations were identified in fragments of human CC, and their frequency depends on ethnicity, etiology, tumor anatomical location and the stage at which it is found [3, 7, 13-15]. Several studies have demonstrated an abnormal expression of KRAS and TP53 being these genetic alterations associated with a more aggressive phenotype of this malignancy. In addition, it has been suggested that, in this type of tumor, alterations in P53 expression may mediate modifications in various intracellular signaling cascades. Other tumor suppressor genes may be inactivated in CC, including P16INK4a, P14ARF, DPC4/ $S M A D 4$ and $A P C$, varying the correlation between these markers and prognosis from study to study $[3,7,13,16]$.

Interleukin-6 (IL-6) seems like a critical signaling molecule in the pathogenesis of various cancers and can be found upstream or downstream of several other oncogenes [17-21]. In this context, the CC is no exception, and IL-6 is a key cytokine in the pathogenesis of this neoplasm being its proliferative effect confirmed (Fig. 1). High serum concentrations of IL-6 were detected in patients with CC, and beyond that, the cells of this type of tumor generally overexpress the gp130 subunit of its receptor. It is also known that of IL-6 production stimulation leads to an increased expression of MCL-1 antiapoptotic protein, which makes CC cells resistant to therapies [3, 7, 22]. It has also been shown in this type of neoplasia that IL-6 induces an increase in telomerase activity, resulting in the inhibition of the telomeres shortening with consequent evasion of cellular senescence. In addition, IL- 6 activates the P44/42 and P38 MAPKs, two key components in the proliferation process. Activated P38 MAPK decreases the cyclin-dependent kinase inhibitor P21, a well-known cell cycle negative regulator. Taking into account the importance that IL-6 has in CC development, the search for targeted therapies to this interleukin appears to be a promising option for $\mathrm{CC}[3,7]$.

There is also a cross talk between IL- 6 and other signaling pathways. It is known, for example, that IL-6 mediates the overexpression of EGFR. Tyrosine kinases receptors, such as EGFR, are overexpressed in CC, as well as in other types of cancer, modulating the tumor biology. Some studies have shown, in this neoplasm, that the inhibition of EGFR signaling pathway could significantly suppress cell growth. In this type of tumor, EGFR 
Fig. 1 Some molecular actions of IL-6 in CC. IL-6 activates P44/42 and P38 MAPKs, mediates EGFR overexpression, induces an increase in telomerase activity and induces an increase in MCL-1 expression
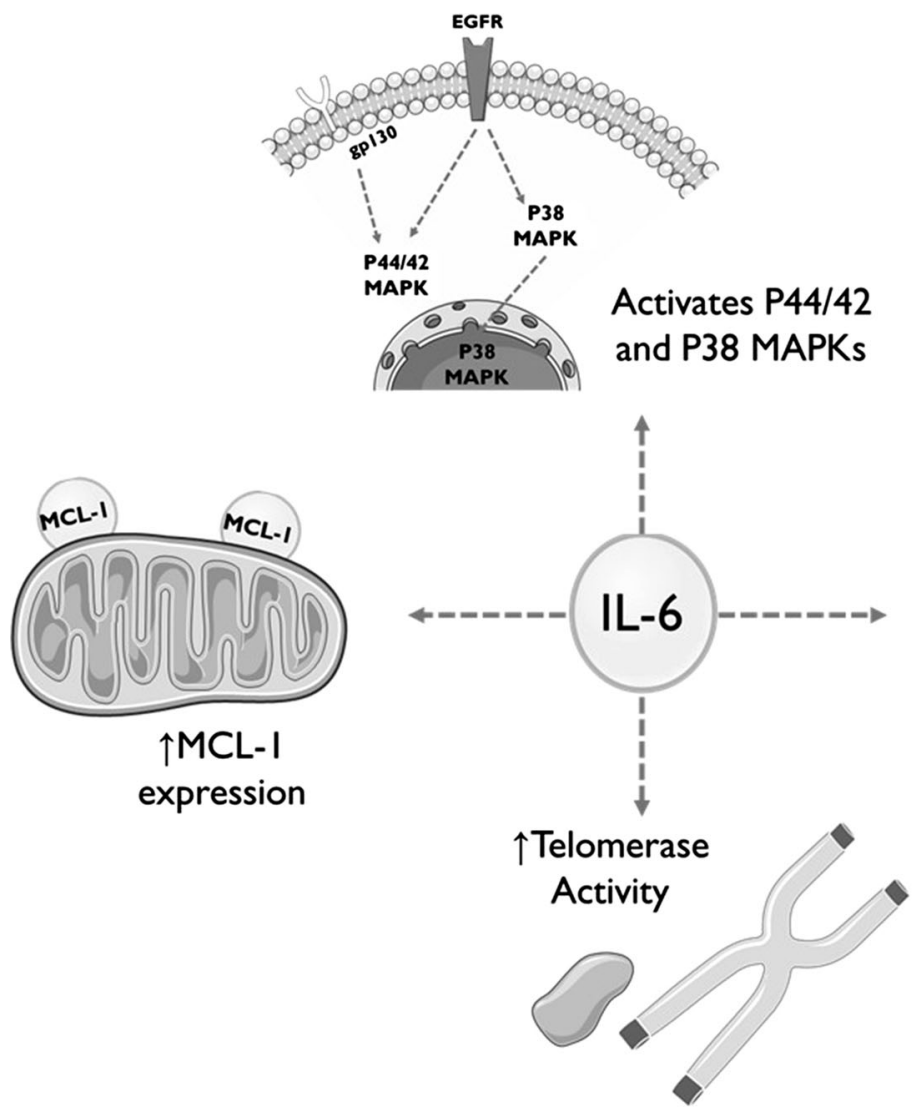

Mediates EGFR overexpression

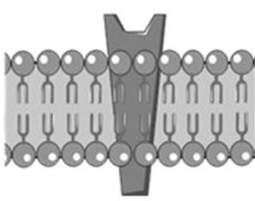

phosphorylation results in the activation of kinases that are downstream in some signaling pathways such as P42/44 MAPK and P38 MAPK which, in turn, increase the COX-2 expression. Still, COX-2 plays an important role in carcinogenesis of $\mathrm{CC}$ by inhibiting the process of apoptosis and stimulation of cell growth [3, 7, 22, 23].

Another molecule that induces COX-2 is the tyrosine kinase erbB-2, which, in CC, is overexpressed and is involved in the carcinogenesis and progression of this type of cancer. The erbB-2 is an EGFR homolog, and its ability to homodimerize or heterodimerize with other members of the EGF family results in activation of the RAF/MAPK signaling pathway $[3,7,22,23]$.

Also hepatocyte growth factor (HGF) and its receptor c-Met are frequently overexpressed in CC [3, 7, 24]. HGF is mitogenic, and the increase in its production, along with the overexpression of its receptor, represents an autocrine mechanism to stimulate cell growth and proliferation. Along with the promotion of cellular signaling pathways that stimulate cell proliferation, there is also in CC a decrease in factors that inhibit cell proliferation. For example, in this neoplasia, the response to TGF- $\beta 1$ is aberrant, resulting in an increase of proliferative index [3, 7].

In the recent years, it was shown in human biopsies of intrahepatic $\mathrm{CC}$ that this type of tumor has increased expression of sodium iodide symporter (NIS), a molecule that mediates the iodine uptake $[25,26]$. These results were then confirmed in in vitro studies, and a recent study in a human extrahepatic $\mathrm{CC}$ cell line shows that these cells also overexpress NIS [27, 28]. NIS has a key role in metabolic radiotherapy, using iodine-131, for the treatment of thyroid tumors, being also currently under active investigation for the treatment of extrathyroidal tumors. This means that NIS overexpression opens a new possibility of treatment for CC [26, 28-31].

On the other hand, a recent study showed that glucose transporter (GLUT-1) expression in CC is correlated with poor prognosis and aggressive behavior, and according to the authors, GLUT-1 might be a therapeutic target for CC [32].

In summary, there is a complex network of different factors and signaling pathways that are involved in $\mathrm{CC}$ development, growth and propagation.

$\mathrm{CC}$ is characterized as a chemo- and radioresistant tumor, which underlies much of the discouraging results obtained with currently available therapies [33, 34]. However, what are the mechanisms leading to this resistance?

Similar to what happens in the majority of cancers, in $\mathrm{CC}$, one of the main reasons that make this type of tumor resistant to therapy is usually its intrinsic resistance to apoptosis. As mentioned above, it is known that most cases of CC have mutations in the TP53 gene; however, the role that this gene and P53 have in the prognosis of this cancer 
is not yet fully understood [16]. Some studies have shown that changes in the TP53 gene or in the P53 protein may, in this type of tumor, lead to changes in the underlying intracellular signaling cascades, thereby inducing resistance to therapies $[16,35]$. Other studies also indicate that $M D M 2$ gene is overexpressed in most cases of $\mathrm{CC}$, which can influence P53 expression and function. Therefore, the inactivation of P53 by MDM2 plays an extremely important role in the resistance to apoptosis [16, 35-37].

In addition to P53, there are several other tumor suppressor genes whose expression is deregulated in CC, such as the case of DPC4/SMAD4 that is underexpressed in most cases of this tumor. On the other hand, P73 that induces inhibition of cell growth and apoptosis through activation of p53-responsive genes seems to be overexpressed in a wide variety of CCs. A loss in DPC4 (deleted in pancreatic carcinoma, locus 4) expression, a tumor suppressor gene that regulates transduction of signals from TGF- $\beta$ superfamily was noted in 55 and $60 \%$ of extrahepatic CCs $[35,38]$.

Besides the evaluation of tumor suppressor genes, in order to clarify the molecular mechanisms that confer resistance to therapy, the gene and protein expression of some members of the BCL-2 family of this neoplasm have also been subject of study. Although the results are sometimes a little contradictory, there are some studies which indicate that the resistance to apoptosis, characteristic of CC, may result from the overexpression of BCL-2, BCL-XL and MCL-1 antiapoptotic proteins [39-41].

One of the other mechanisms that make the tumors resistant to cell death and, consequently, to therapy is the expression of transmembrane proteins such as the efflux proteins of $\mathrm{ABC}$ family [42, 43]. This is also a common characteristic in the primary liver tumors such as HCC and CC [34, 44-47]. The majority of the cases of CC express P-glycoprotein (PGP), and it was shown that the overexpression of this protein induces resistance to drugs used in chemotherapy. In other words, also in CC the response to chemotherapy is inversely correlated with PGP expression [45, 46]. Likewise, overexpression of MRP1 (multidrug resistance-related protein-1), another protein of the $A B C$ family of transporters, is associated with chemoresistance in primary liver tumors and consequent poor prognosis [48, 49]. Consistent with this, a 2005 study of Tepsiri and colleagues that evaluated in five human CC cell lines, the expression of several genes involved in multidrug resistance, found that all of them express MRP1 [50].

\section{Diagnosis and treatment}

In most cases, $\mathrm{CC}$ is a silent tumor, and symptoms only occur in advanced stages of the disease. In addition, the clinical signs are dependent on the location of the tumor and the growth pattern. Most patients with extrahepatic CC present biliary tract obstruction with painless obstructive jaundice, sometimes associated with cholangitis. In turn, the intrahepatic formations present late symptoms, typical of hepatic malignant, such as weight loss, anorexia and abdominal pain $[3,11]$.

In terms of diagnosis, there are many studies, either invasive or noninvasive, that can be used. Some serum tumor markers, although not being specific to $\mathrm{CC}$, can provide important information, especially in patients with underlying PSC. In this sense, the most commonly used tumor markers are carcinoembryonic antigen (CEA) and cancer antigen 19-9 (CA 19-9). The presence of both may be increased in $\mathrm{CC}$, although its employment alone is questionable. However, recent studies have shown that the combination of these two markers may be useful for the diagnosis and management of CC [5, 11, 51].

Imaging studies are essential for diagnosis and staging of this type of tumor, and there are several imaging modalities that can provide useful information when there is suspicion of CC. Usually, as an initial approach, patients with jaundice undergo an abdominal ultrasound which, although being a dependent operator technique, is a very sensitive method for bile duct visualization. Computed tomography (CT) is typically used to establish the lesion location as well as to define the local spread and metastization. The use of this imaging technique in addition to contrast is a quite sensitive method for the detection of tumors of the biliary tract, also allowing to determine the level of biliary obstruction and the presence of liver's atrophy $[3,5,9]$.

Besides this technique, the magnetic resonance cholangiopancreatography (MRCP) may also be used. This modality uses nuclear magnetic resonance to create a threedimensional image of the biliary tree, hepatic parenchyma and vascular structures which is comparable to retrograde endoscopic cholangiopancreatography in the detection of malignant lesions in the biliary tract. An advantage of MRCP is that it can identify the luminal involvement, providing better information about the stage of the tumor. This classification is extremely important, especially in determining the resectability of the tumor $[5,9,11]$.

Positron emission tomography (PET) using ${ }^{18} \mathrm{~F}$-FDG has also shown a great sensitivity in the diagnosis of this type of tumors. However, we must not forget that in the inflammatory processes of the biliary tract, as for example in patients with PSC, PET can provide false positives, which limits the use of this methodology. However, this imaging technique remains an extremely important tool in the detection of distant metastasis as well as recurrence after resection $[5,11]$.

It was already mentioned that most cases of $\mathrm{CC}$ are diagnosed in an advanced stage of the disease, when this 
type of tumor is considered to be devastating, having the patients a median survival of $<24$ months $[5,6]$. The prognosis is, therefore, extremely poor, and survival rate is $5-10 \%[5,6]$. Surgical treatments are the only ones that offer curative options; however, due to the late diagnosis, these are not applicable to the vast majority of patients [5, $11,52]$. In the case of surgical resection of $\mathrm{CC}$, this aims to perform a complete excision with negative margins. There are, however, four different factors that determine the possibility of performing a resection by partial hepatectomy. They are the tumor extension within the biliary tree, the vascular invasion, the lobar hepatic atrophy and the metastatic disease. Although hepatic resection is considered as the only curative therapy, the survival rate after surgery where resection is considered successful is only of $25-30 \%$ [5, 11]. Liver transplantation as a therapeutic alternative in CC is controversial. Due to the high recurrence rate reported by several authors, some institutions have abandoned this therapeutic strategy for this type of tumor. Nevertheless, some successful cases have been described $[5,11]$.

Considering that the vast majority of CCs at diagnosis are unresectable, patients are subjected to other therapeutic modalities with minimal success $[5,11,53]$. Therefore, chemotherapy is only used to control the disease and improve the quality of life in patients with unresectable CC, in patients with tumor recurrence and metastatic CC. The chemotherapeutic agents most widely used in the treatment of $\mathrm{CC}$ are 5-fluorouracil and gemcitabine either alone or in combination with other drugs. These cytostatics have been associated with a wide variety of other drugs such as cisplatin, oxaliplatin, docetaxel, paclitaxel, mitomycin-C, doxorubicin, epirubicin and interferon- $\alpha$; however, all combinations tested until now showed low response rates, and there is not currently any randomized study which shows a clear benefit of any chemotherapeutic regimen in this type of tumors. When comparing the survival time, after 5 years, of patients who underwent only to surgery or a combined regime of surgery and chemotherapy, there were no significant differences, which makes urgent to find pharmacological alternatives to assist in the treatment of this neoplasia [3, 5, 54].

The use of radiotherapy, either external or brachytherapy, for CC treatment has been explored; however, benefits have not been observed. Nevertheless, some studies indicate that patients with positive surgical margins can benefit from adjuvant radiotherapy. However, the role of this type of treatment in resected patients with negative margins is less clear, and there are studies demonstrating no occurrence of benefits $[5,9,11]$. Due to these disappointing results, the local recurrence after surgical resection is usual and many authors have proposed the use of radiotherapy, either alone or in combination with chemotherapy (chemoradiotherapy), as a local control strategy. Given the radiosensitizer potential of 5-fluorouracil, theoretically, it would be expected that the use of this drug in combination with radiotherapy was more effective than any of the therapeutic approaches alone. However, and although there are no randomized studies of this combination therapy, the retrospective analysis shows no increase in survival as compared to radiotherapy alone $[5,9,55]$.

Once the use of the available therapeutic strategies has shown completely disappointing results, it is imperative to search for new molecules and therapeutic targets to combat this neoplasia. Some drugs that act on specific signaling pathways, essential for the pathogenesis of $\mathrm{CC}$, are already approved for clinical use in other types of cancer. These include EGFR inhibitors (gefitinib, cetuximab and erlotinib), RAF kinase inhibitors (sorafenib), VEGF-directed inhibitors (bevacizumab and sorafenib) and HER-2-directed inhibitors (lapatinib and trastuzumab) $[3,56]$. There are already some clinical trials which aim at evaluating the effect of celecoxib, a COX-2 inhibitor, and the tyrosine kinase receptor inhibitors, sorafenib, erlotinib and bevacizumab alone or in combination with other drugs for the treatment of CC [3, 56]. Another strategy that has been considered is the sensitization of the CC cells to apoptosis mediated by TRAIL through MCL-1 downregulation using, for example, sorafenib. These are some approaches of targeted therapies that can be applied to the treatment of CC. The promising role that sorafenib has for acting in several areas simultaneously, i.e., being an inhibitor of RAF kinase, VEGFR, tyrosine kinase receptors as well as be able to induce the apoptosis mediated by TRAIL $[3,56]$, should be noted.

Although in clinical practice sorafenib is not used in CC treatment, currently there are several studies which indicate that this drug has a promising role in the CC treatment [5760]. Mutations in the MAPK signaling pathway are one of the most common genetic alterations in $\mathrm{CC}$, present in approximately $60 \%$ of cases of human tumors. Within this signaling pathway, mutations in the RAS are described in about $56 \%$ of these tumors and B-RAF mutations in about $22 \%$, which makes sorafenib a promising therapeutic weapon for CC treatment [57]. Indeed, there have been already performed in vitro studies that emphasize the potential effect of sorafenib on CC therapy. In 2007, Huther et al. demonstrated that this drug inhibits the proliferation of human CC cell lines in a time- and dosedependent manner. This antiproliferative effect was correlated with the induction of cell death by apoptosis and cell cycle arrests in the G0/G1 phase [57]. In this experimental work, the authors were not able to draw more accurate conclusions about the mechanisms by which sorafenib induced the cell cycle's blockade and apoptosis; however, in 2005, another study had already provided some 
useful information [61]. In fact, in this study, conducted in several tumor cell lines, it was found that in the CC cells, a downregulation of antiapoptotic MCL-1 protein occurred in response to sorafenib [61]. Later, in 2011, Sugiyama et al. tested sorafenib in eight human CC cell lines and also performed in vivo studies. From the in vitro studies, the treatment with sorafenib led to inhibition of cell proliferation and induction of apoptosis through the inhibition of the RAF/MEK/MAPK signaling pathway, reduction in phosphorylation and consequent activation of STAT3 and downregulation of MCL-1. In in vivo studies, the authors found that oral administration of sorafenib significantly inhibited tumor growth of heterotopic xenografts [58]. Other in vitro study has shown the existence of synergy in the combined use of sorafenib with doxorubicin and gemcitabine in CC treatment [62].

In 2011, a 70-year-old male patient diagnosed with unresectable intrahepatic $\mathrm{CC}$ and no indication for any treatment regimen was treated with $400 \mathrm{mg}$ of sorafenib daily. It was found that this treatment led to a significant improvement of symptoms, improving liver function and a decrease in the levels of tumor markers. The patient started the treatment in March 2008 and in March 2010 and was still alive presenting some quality of life [63].

In 2013, another case was reported. It was a 51-year-old patient diagnosed with CC. After diagnosis, the patient began systemic chemotherapy and several chemotherapeutic schemes have been tested, such as GEMOX and the association of capacitabine with 5-fluorouracil, but the disease continued to progress. At this point, sorafenib became the fourth-line agent managing to extend patient life for four more years [59].

Thus, although the use of sorafenib for the treatment of $\mathrm{CC}$ is not yet been adopted, some evidence suggest that the use of this drug in the treatment of $\mathrm{CC}$ may have a promising future.

\section{Conclusions}

Nowadays, the treatment of CC is only available for a very small number of patients because this tumor is asymptomatic in the early stages of the disease. Actually, in the majority of the cases, at the time of diagnosis, the patients can no longer take advantage of the surgical options which are currently the only chance of cure. Since this kind of tumor is highly radio- and chemoresistant, conventional therapies such as chemotherapy and radiotherapy are generally used only with palliative purposes. Concerning this type of tumor, it has several genetic alterations that can explain, at least in part, the high resistance to radiotherapy and chemotherapy. In this context, changes in the expression of KRAS and TP53 genes should be highlighted.
Changes in IL- 6 production and in the expression of some transporters such as GLUT-1 and NIS as well as the proteins of the BCL-2 family should also be mentioned. Since this tumor incidence has grown markedly in recent years and the treatment options are minor, it is urgent to investigate new therapeutic approaches to combat it.

Acknowledgments Ana Filipa Brito would like to thank the Portuguese Foundation for Science and Technology for the award of a $\mathrm{PhD}$ scholarship (SFRH/BD/61378/2009). The authors thank to Center of Investigation on Environmental, Genetics and Oncobiology by the financing of the project 09/12. Support: Portuguese Foundation for Science and Technology, Portugal (Strategic Project PEst-C/SAU/ UI3282/2013 and UID/NEU/04539/2013), COMPETE-FEDER.

\section{Compliance with ethical standards}

Conflict of interest The authors declare that they have nothing to disclose.

\section{References}

1. Mao K, Jiang W, Liu J, Wang J. Incidence of subsequent cholangiocarcinomas after another malignancy: trends in a population-based study. Medicine (Baltimore). 2015;94:e596.

2. Yu MC, Yuan JM. Environmental factors and risk for hepatocellular carcinoma. Gastroenterology. 2004;127(5 Suppl 1):S72-8.

3. Blechacz B, Gores GJ. Cholangiocarcinoma: advances in pathogenesis, diagnosis, and treatment. Hepatology. 2008;48:308-21.

4. Cardinale V, Bragazzi MC, Carpino G, Torrice A, Fraveto A, Gentile R, et al. Cholangiocarcinoma: increasing burden of classifications. Hepatobiliary Surg Nutr. 2013;2:272-80.

5. Anderson CD, Pinson CW, Berlin J, Chari RS. Diagnosis and treatment of cholangiocarcinoma. Oncologist. 2004;9:43-57.

6. Goodman ZD. Neoplasms of the liver. Mod Pathol. 2007; 20(Suppl 1):S49-60.

7. Al-Bahrani R, Abuetabh Y, Zeitouni N, Sergi C. Cholangiocarcinoma: risk factors, environmental influences and oncogenesis. Ann Clin Lab Sci. 2013;43:195-210.

8. Bragazzi MC, Cardinale V, Carpino G, Venere R, Semearo R, Gentile R, et al. Cholangiocarcinoma: epidemiology and risk factors. Transl Gastrointest Cancer. 2012;1:21-32.

9. Aljiffry M. Advances in diagnosis, treatment and palliation of cholangiocarcinoma: 1990-2009. World J Gastroenterol. 2009; 15(34):4240-62.

10. Chapman RW. Risk factors for biliary tract carcinogenesis. Ann Oncol. 1999;10(Suppl 4):308-11.

11. Friman S. Cholangiocarcinoma: current treatment options. Scand J Surg. 2011;100(1):30-4.

12. Ralphs $\mathrm{S}$, Khan $\mathrm{S}$. The role of hepatitis viruses in cholangiocarcinoma. J Viral Hepat. 2013;20(5):297-305.

13. O’Dell MR, Huang JL, Whitney-Miller CL, Deshpande V, Rothberg P, Grose V, et al. Kras(G12D) and p53 mutation cause primary intrahepatic cholangiocarcinoma. Cancer Res. 2012; 72(6):1557-67.

14. Maroni L, Pierantonelli I, Banales JM, Benedetti A, Marzioni M. The significance of genetics for cholangiocarcinoma development. Ann Transl Med. 2013;1(3):28.

15. Patel T. New insights into the molecular pathogenesis of intrahepatic cholangiocarcinoma. J Gastroenterol. 2014;49(2):165-72.

16. Khan SA, Thomas HC, Toledano MB, Cox IJ, Taylor-Robinson SD. p53 Mutations in human cholangiocarcinoma: a review. Liver Int. 2005;25(4):704-16. 
17. Fava G, Lorenzini I. Molecular pathogenesis of cholangiocarcinoma. Int J Hepatol. 2012;2012:630543.

18. Johnson C, Han Y, Hughart N, McCarra J, Alpini G, Meng F. Interleukin- 6 and its receptor, key players in hepatobiliary inflammation and cancer. Transl Gastrointest Cancer. 2012;1(1):58-70.

19. Waldner MJ, Foersch S, Neurath MF. Interleukin-6-a key regulator of colorectal cancer development. Int J Biol Sci. 2012; 8(9):1248-53.

20. Wei LH, Kuo ML, Chen CA, Chou CH, Lai KB, Lee CN, et al. Interleukin- 6 promotes cervical tumor growth by VEGF-dependent angiogenesis via a STAT3 pathway. Oncogene. 2003;22(10): 1517-27.

21. Xu H, Hu MB, Bai PD, Zhu WH, Liu SH, Hou JY, et al. Proinflammatory cytokines in prostate cancer development and progression promoted by high-fat diet. Biomed Res Int. 2015;2015:249741.

22. Wehbe H, Henson R, Meng F, Mize-Berge J, Patel T. Interleukin6 contributes to growth in cholangiocarcinoma cells by aberrant promoter methylation and gene expression. Cancer Res. 2006; 66(21):10517-24.

23. Tsubouchi H. Sustained activation of epidermal growth factor receptor in cholangiocarcinoma: a potent therapeutic target? J Hepatol. 2004;41(5):859-61.

24. Socoteanu MP, Mott F, Alpini G, Frankel AE. c-Met targeted therapy of cholangiocarcinoma. World J Gastroenterol. 2008; 14(19):2990-4.

25. Kim JH, Han SY, Lee SW, Baek YH, Kim HY, Kim JH, et al. Sodium iodide symporter and phosphatase and tensin homolog deleted on chromosome ten expression in cholangiocarcinoma analysis with clinicopathological parameters. Gut Liver. 2012; 6(3):374-80.

26. Liu B, Hervé J, Bioulac-Sage P, Valogne Y, Roux J, Yilmaz F, et al. Sodium iodide symporter is expressed at the preneoplastic stages of liver carcinogenesis and in human cholangiocarcinoma. Gastroenterology. 2007;132(4):1495-503.

27. Guerrieri F, Piconese S, Lacoste C, Schinzari V, Testoni B, Valogne Y, et al. The sodium/iodide symporter NIS is a transcriptional target of the p53-family members in liver cancer cells. Cell Death Dis. 2013;4:e807.

28. Ribeiro AC, Fernandes AI, Brito AF, Abrantes AM, Laranjo M, Santos K, et al. Iodine-131: an option for the cholangiocarcinoma treatment? EJNMMI. 2014;41:S640.

29. Filetti S, Bidart JM, Arturi F, Caillou B, Russo D, Schlumberger M. Sodium/iodide symporter: a key transport system in thyroid cancer cell metabolism. Eur J Endocrinol. 1999;141(5):443-57.

30. Mishra A, Pal L, Mishra SK. Distribution of Na +/I- symporter in thyroid cancers in an iodine-deficient population: an immunohistochemical study. World J Surg. 2007;31(9):1737-42.

31. Kogai T, Taki K, Brent GA. Enhancement of sodium/iodide symporter expression in thyroid and breast cancer. Endocr Relat Cancer. 2006;13(3):797-826.

32. Kubo Y, Aishima S, Tanaka Y, Shindo K, Mizuuchi Y, Abe K, et al. Different expression of glucose transporters in the progression of intrahepatic cholangiocarcinoma. Hum Pathol. 2014; 45(8):1610-7.

33. Macias RIR. Cholangiocarcinoma: biology, clinical management, and pharmacological perspectives. ISRN Hepatol. 2014;2014:1-13.

34. Marin JJ, Lozano E, Briz O, Al-Abdulla R, Serrano MA, Macias RI. Molecular bases of chemoresistance in cholangiocarcinoma. CurrDrug Targets. 2015; [Epub ahead of print].

35. Briggs CD, Neal CP, Mann CD, Steward WP, Manson MM, Berry DP. Prognostic molecular markers in cholangiocarcinoma: a systematic review. Eur J Cancer. 2009;45(1):33-47.

36. Horie S, Endo K, Kawasaki H, Terada T. Overexpression of MDM2 protein in intrahepatic cholangiocarcinoma: relationship with p53 overexpression, Ki-67 labeling, and clinicopathological features. Virchows Arch. 2000;437(1):25-30.

37. Wang J, Wang X, Xie S, Yan Z, Li Z, Li Y, et al. P53 status and its prognostic role in extrahepatic bile duct cancer: a meta-analysis of published studies. Dig Dis Sci. 2011;56(3):655-62.

38. Lee KT, Chang WT, Wang SN, Chuang SC, Chai CY, Hu SW. Expression of DPC4/Smad4 gene in stone-containing intrahepatic bile duct. J Surg Oncol. 2006;94(4):338-43.

39. Harnois DM, Que FG, Celli A, LaRusso NF, Gores GJ. Bcl-2 is overexpressed and alters the threshold for apoptosis in a cholangiocarcinoma cell line. Hepatology. 1997;26(4):884-90.

40. Okaro AC, Deery AR, Hutchins RR, Davidson BR. The expression of antiapoptotic proteins $\mathrm{Bcl}-2, \mathrm{Bcl}-\mathrm{X}(\mathrm{L})$, and $\mathrm{Mcl}-1$ in benign, dysplastic, and malignant biliary epithelium. J Clin Pathol. 2001;54(12):927-32.

41. Okaro AC, Fennell DA, Corbo M, Davidson BR, Cotter FE. Pk11195, a mitochondrial benzodiazepine receptor antagonist, reduces apoptosis threshold in Bcl-X. Gut. 2002;51(4):556-61.

42. Casalta-lopes J, Abrantes AM, Laranjo M, Rio J, Gonçalves AC, Oliveiros B, et al. Efflux pumps modulation in colorectal adenocarcinoma cell lines : the role of nuclear medicine. J Cancer Ther. 2011;2(3):408-17.

43. Gottesman MM, Fojo T, Bates SE. Multidrug resistance in cancer: role of ATP-dependent transporters. Nat Rev Cancer. 2002;2(1):48-58.

44. Chen YB, Yan ML, Gong JP, Xia RP, Liu LX, Li N, et al. Establishment of hepatocellular carcinoma multidrug resistant monoclone cell line HepG2/mdr1. Chin Med J (Engl). 2007; 120(8):703-7.

45. Itsubo M, Ishikawa $T$, Toda G, Tanaka M. Immunohistochemical study of expression and cellular localization of the multidrug resistance gene product P-glycoprotein in primary liver carcinoma. Cancer. 1994;73(2):298-303.

46. Seo S, Hatano E, Higashi T, Nakajima A, Nakamoto Y, Tada M, et al. Fluorine-18 fluorodeoxyglucose positron emission tomography predicts lymph node metastasis, P-glycoprotein expression, and recurrence after resection in mass-forming intrahepatic cholangiocarcinoma. Surgery. 2008;143(6):769-77.

47. Shen DW, Lu YG, Chin KV, Pastan I, Gottesman MM. Human hepatocellular carcinoma cell lines exhibit multidrug resistance unrelated to MRD1 gene expression. J Cell Sci. 1991;98(Pt 3): 317-22.

48. Borst P, Evers R, Kool M, Wijnholds J. The multidrug resistance protein family. Biochim Biophys Acta. 1999;1461(2):347-57.

49. Zahreddine H, Borden KLB. Mechanisms and insights into drug resistance in cancer. Front Pharmacol. 2013;4:28.

50. Tepsiri N, Chaturat L, Sripa B, Namwat W, Wongkham S, Bhudhisawasdi V, et al. Drug sensitivity and drug resistance profiles of human intrahepatic cholangiocarcinoma cell lines. World J Gastroenterol. 2005;11(18):2748-53.

51. Malaguarnera G, Paladina I, Giordano M, Malaguarnera M, Bertino G, Berretta M. Serum markers of intrahepatic cholangiocarcinoma. Dis Markers. 2013;34(4):219-28.

52. Razumilava N, Gores GJ. Cholangiocarcinoma. Lancet. 2014; 383(9935):2168-79.

53. Razumilava N, Gores GJ. Classification, diagnosis, and management of cholangiocarcinoma. Clin Gastroenterol Hepatol. 2013;11(1):13-21.

54. Thongprasert S. The role of chemotherapy in cholangiocarcinoma. Ann Oncol. 2005;16(Suppl 2):ii93-6.

55. Bonet Beltrán M, Roth AD, Mentha G, Allal AS. Adjuvant radiochemotherapy for extrahepatic biliary tract cancers. BMC Cancer. 2011;11:267.

56. Noel MS, Hezel AF. New and emerging treatment options for biliary tract cancer. Onco Targets Ther. 2013;6:1545-52. 
57. Huether A, Höpfner M, Baradari V, Schuppan D, Scherübl H. Sorafenib alone or as combination therapy for growth control of cholangiocarcinoma. Biochem Pharmacol. 2007;73(9):1308-17.

58. Sugiyama H, Onuki K, Ishige K, Baba N, Ueda T, Matsuda S, et al. Potent in vitro and in vivo antitumor activity of sorafenib against human intrahepatic cholangiocarcinoma cells. J Gastroenterol. 2011;46(6):779-89.

59. Chakunta HR, Sunderkrishnan R, Kaplan MA, Mostofi R. Cholangiocarcinoma: treatment with sorafenib extended life expectancy to greater than four years. J Gastrointest Oncol. 2013;4(4):E30-2.

60. Kim do H, Jeong YI, Chung CW, Kim CH, Kwak TW, Lee HM, et al. Preclinical evaluation of sorafenib-eluting stent for suppression of human cholangiocarcinoma cells. Int $\mathbf{J}$ Nanomed. 2013;8:1697-711.
61. Yu C, Bruzek LM, Meng XW, Gores GJ, Carter CA, Kaufmann $\mathrm{SH}$, et al. The role of Mcl-1 downregulation in the proapoptotic activity of the multikinase inhibitor BAY 43-9006. Oncogene. 2005;24(46):6861-9.

62. Pignochino Y, Sarotto I, Peraldo-Neia C, Penachioni JY, Cavalloni G, Migliardi G, et al. Targeting EGFR/HER2 pathways enhances the antiproliferative effect of gemcitabine in biliary tract and gallbladder carcinomas. BMC Cancer. 2010;10:631.

63. Pinter M, Sieghart W, Reisegger M, Wrba F, Peck-Radosavljevic M. Sorafenib in unresectable intrahepatic cholangiocellular carcinoma: a case report. Wien Klin Wochenschr. 2011;123(1-2):61-4. 